\title{
Complementation of Xanthobacter Py2 mutants defective in epoxyalkane degradation, and expression and nucleotide sequence of the complementing DNA fragment
}

\author{
Jelto Swaving, Carel A. G. M. Weijers, Albert J. J. van Ooyen and \\ Jan A. M. de Bont
}

Author for correspondence: Jelto Swaving. Tel: +31 837083393 . Fax: + 31837084978. e-mail: Jelto.Swaving@algemeen.im.wau.nl

Division of Industrial Microbiology, Department of Food Science, Agricultural University, PO Box 8129, 6700 EV Wageningen, The Netherlands

\begin{abstract}
Three Xanthobacter Py2 mutants (M3, M8 and M10) lacking epoxyalkanedegrading activity were isolated and characterized. All mutants were able to grow on acetone, the degradation product of 1,2-epoxypropane conversions. Furthermore, they contained the unidentified 'low molecular mass fraction' (LMF) necessary for epoxyalkane-degrading activity. Three cosmids from a gene bank complemented the mutation in M10 and M8 but not in mutant M3. Epoxyalkane-degrading activity in crude extracts of 1,2-epoxypropane-grown complemented mutants was similar to the wild-type activity. Surprisingly, M10 transformed with complementing cosmid pEP9 showed a constitutively expressed epoxyalkane-degrading activity, which was not observed in the wild-type strain. The cosmid PEP9 was conjugated into Xanthobacter autotrophicus GJ10, which is not able to degrade 1,2-epoxypropane. In crude extracts of $X$. autotrophicus GJ10(pEP9), epoxyalkane-degrading activity was demonstrated, but only after the addition of the LMF from Xanthobacter Py2. Hybridization experiments demonstrated an overlap on complementing cosmids PEP1, pEP3 and pEP9. Subcloning revealed a $4.8 \mathrm{~kb}$ EcoRI-HindIII fragment to be necessary for complementing the mutant M10. In the sequence of this fragment four different ORFs were found.
\end{abstract}

Keywords: Xanthobacter Py2, epoxyalkane degradation, complementation

\section{INTRODUCTION}

Several methods have been described to produce optically pure epoxides by biological methods (Weijers et al., 1988b; Leak et al., 1992; De Bont, 1993). One such method is the enantioselective degradation of racemic epoxides; however, such a process yields a maximum of $50 \%$ product. Xanthobacter Py 2 was isolated on propene as sole carbon and energy source (Van Ginkel \& De Bont, 1986). This strain is able to degrade the (2S)-forms of trans-2,3-epoxybutane and 2,3-epoxypentane, resulting in optically pure (2R)-2,3-epoxyalkanes; the $\mathrm{C}_{3}-\mathrm{C}_{6} 1,2$ epoxyalkanes are degraded completely (Weijers et al., 1988a).

Abbreviation: LMF, low molecular mass fraction.

The EMBL accession number for the sequence reported in this paper is X79863.
Ketones have been identified as degradation products of epoxyalkane metabolism in crude extracts of propenegrown cells (Fig. 1). It has been demonstrated that both NAD and an unidentified 'low molecular mass fraction' (LMF) are involved in the reaction. In a reaction mechanism proposed by Weijers et al. (1994) it was suggested that the LMF is involved in the reduction of the epoxyalkanes to secondary alcohols since the LMF can be replaced by reducing compounds like DTT and other dithiol compounds. The presence of NAD is necessary for the oxidation of the alcohols to the corresponding ketones.

Attempts to purify the epoxyalkane-degrading enzyme have not been successful. Consequently, we have now investigated the genetics of epoxyalkane degradation in Xanthobacter Py2. Eventually, we hope to be able to obtain the enzyme to study further its reaction mechanism.

In this study we report the selection and characterization 


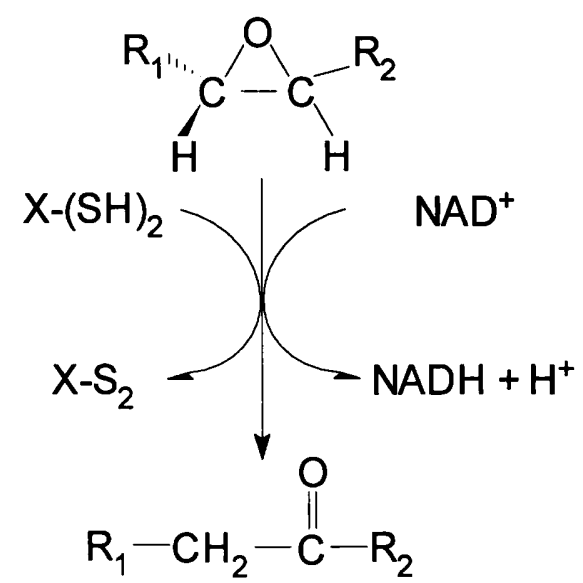

Fig. 1. Proposed degradation pathway of epoxyalkanes by Xanthobacter Py2. X-(SH) 2 represents the LMF necessary for epoxyalkane degradation (Weijers et al., 1994).

of mutants devoid of epoxyalkane-degrading activity, the complementation of these mutants and the expression of one of the complementing cosmids in Xanthobacter autotropbicus GJ10. In addition, a $4.8 \mathrm{~kb}$ fragment complementing the M10 mutation was sequenced and analysed.

\section{METHODS}

General methods. Strains and plasmids used in this study are listed in Table 1. Escherichia coli cells were grown on LB medium (Sambrook et al., 1989). Xantbobacter strains were grown at $30{ }^{\circ} \mathrm{C}$ on yeast extract-glucose medium $\left(10 \mathrm{~g} \mathrm{l}^{-1}\right.$ each) or in sealed flasks with mineral medium (Wiegant \& De Bont, 1980) to which $5 \mathrm{mM}$ volatile compound was added to the water phase or $5 \%(\mathrm{v} / \mathrm{v})$ propene added to the gas phase. For induction of epoxyalkane-degrading activity, cells were grown in yeast extract-glucose medium $(750 \mathrm{ml})$ in sealed 51 flasks to which $1 \%$ propene was added to the gas phase as inducing agent. Cells cultivated on plates were placed in a desiccator when a volatile compound was used as a substrate; for each plate $(20 \mathrm{ml})$ $0.1 \mathrm{mmol}$ of the substrate was added to give a final concentration of $5 \mathrm{mM}$. The antibiotics used for the selection of plasmids were tetracycline $\left(12.5 \mathrm{mg} \mathrm{m}^{-1}\right.$ ), ampicillin and kanamycin (both $50 \mathrm{mg} \mathrm{ml}^{-1}$ ). Preparation of crude extracts, and LMF, and the activity assay for epoxyalkane degradation in which the initial degradation rate of 1,2-epoxypropane in extracts is followed using head-space GC analysis, have been described by Weijers et al. (1994).

Mutant isolation. Exponentially growing cultures $(5 \mathrm{ml})$ of Xantbobacter Py 2 grown on propene were UV-irradiated until less than $1 \%$ of the cells survived. The cells were placed in the dark in $20 \mathrm{ml}$ liquid mineral medium with $5 \mathrm{mM}$ acetone until growth was visible. The cells were harvested by centrifugation at $15000 \mathrm{~g}$ for $5 \mathrm{~min}$, washed and resuspended in the same volume of mineral medium containing 1,2-epoxypropane. Glycine $(2 \%, \mathrm{w} / \mathrm{v})$ was added after $8 \mathrm{~h}$ incubation to kill growing cells. After overnight incubation in the glycinecontaining medium, the cells were harvested and washed in sterile demineralized water. Recovery, starvation and killing of the cells was repeated once more before spreading them on yeast extract-glucose plates.

Gene bank preparation. Total DNA was prepared from propene-grown wild-type Xanthobacter Py2 cells. Cells from $750 \mathrm{ml}$ cultures were harvested, resuspended in $25 \mathrm{ml}$ TEG (10 mM Tris, $1 \mathrm{mM}$ EDT A, $50 \mathrm{mM}$ glucose, $\mathrm{pH} 8.0$ ) and lysed by the addition of SDS $(2 \%, \mathrm{w} / \mathrm{v}$, final concentration). Proteins were removed by extraction with phenol/chloroform, and DNA was precipitated with 2-propanol. Purified DNA was dissolved in TE buffer, partially digested with Sau3A and dephosphorylated. The two pLAFR 5 cosmid arms were prepared by digestion with ScaI and BamHI (Keen et al., 1988). Ligated DNA was packaged using the Packagene in vitro packaging system supplied by Promega and used to transduce E. coli LE392; the transduced cells were spread on plates (100-200 colonies per plate). After overnight growth cells were resuspended and pooled in LB supplemented with $30 \%(\mathrm{v} / \mathrm{v})$ glycerol and stored at $-80^{\circ} \mathrm{C}$.

Triparental mating. This was carried out essentially as described by Janssen et al. (1989) but instead of NB agar, yeast extract-

Table 1. Bacterial strains and cosmids used

\begin{tabular}{|c|c|c|}
\hline Organism or cosmid & Relevant properties & Reference \\
\hline \multicolumn{3}{|l|}{ E. coli } \\
\hline LE392 & $\operatorname{RecA}^{-}$ & Sambrook et al. (1989) \\
\hline TG1 & $\operatorname{Rec}^{-}$ & Sambrook et al. (1989) \\
\hline HB101 & $\operatorname{RecA}^{-}$ & Boyer \& Roulland-Dussoix (1969) \\
\hline \multicolumn{3}{|l|}{ Xanthobacter } \\
\hline Py2 & Epoxyalkane-using wild-type & Van Ginkel \& De Bont (1986) \\
\hline Py2 M3 & Defective in epoxyalkane degradation & This study \\
\hline Py2 M8 & Defective in epoxyalkane degradation & This study \\
\hline Py2 M10 & Defective in epoxyalkane degradation & This study \\
\hline$X$. autotrophicus GJ10 & Growth on acetone, not on epoxyalkanes & Janssen et al. (1985) \\
\hline \multicolumn{3}{|l|}{ Cosmids } \\
\hline pLAFR5 & $\mathrm{Tc}^{\mathrm{r}} \mathrm{RK} 2$ replicon $m o b^{+}$ & Keen et al. (1988) \\
\hline pEP1, $-3,-9$ & pLAFR5 containing Xantbobacter Py2 DNA & This study \\
\hline pRK2013 & $\operatorname{tra}$ (RK2) ColE1 replicon $\mathrm{Km}^{\mathrm{r}}$ & Figurski \& Helinski (1979) \\
\hline $\mathrm{pT} 7 / \mathrm{T} 3-19$ & Ap ${ }^{r}$ pUC19 derivative & Life Technologies \\
\hline
\end{tabular}


glucose agar was used. The conjugated recipient strains were selected on tetracycline-containing mineral medium agar plates with either 1,2-epoxypropane or 2-propanol as substrate.

DNA manipulations, Southern transfer, hybridization and nucleotide sequencing. Standard recombinant DNA techniques were performed as described by Sambrook et al. (1989). DNA was isolated from agarose gel using Geneclean II (Bio 101). For Southern transfer, digested cosmid DNA was separated on a $0.7 \%$ agarose gel, blotted onto a nylon membrane (Nytran NY 13N; Schleicher \& Schuell) by capillary transfer and fixed on the membrane by UV-irradiation for $2 \mathrm{~min}$. Probes were prepared using the DIG DNA labelling and detection kit (Boehringer). Hybridization and detection were performed using the same kit, except that the hybridization temperature was $71^{\circ} \mathrm{C}$. A $4.8 \mathrm{~kb}$ EcoRI-HindIII fragment was ligated into plasmid pT7/T3a-19. The insert was sequenced in both directions by Pharmacia Biotech Benelux using the primer walking method. Nucleotide sequencing was performed using the dideoxy chain-termination method (Sanger et al., 1977) using 5'-FITC-labelled primers. Computer analysis of the sequence was accomplished with the Staden program.

\section{RESULTS}

\section{Isolation of Xanthobacter Py2 mutants devoid of epoxyalkane-degrading activity}

The complementation method used for the isolation of Xanthobacter Py2 DNA sequences involved in epoxyalkane degradation by Xanthobacter Py 2 requires mutants defective in the protein responsible for epoxyalkane degradation. To ensure that only this protein is mutated and not enzymes further down the degradation pathway, these mutants should still be able to grow on acetone, the first detectable product of 1,2-epoxypropane degradation. These mutants should also contain the unidentified 'low molecular mass fraction' (LMF) required for epoxide degradation.

Mutants were obtained as described in Methods. Single colonies were streaked on mineral medium plates and incubated for 1 week in a desiccator containing 1,2epoxypropane. Wild-type cells able to grow on 1,2epoxypropane formed large slimy colonies while mutant cells formed very small colonies. Of 450 putative mutants tested, 23 did not grow on 1,2-epoxypropane. These small colonies were sub-cultured twice on mineral medium plates with 1,2-epoxypropane as substrate. This procedure resulted in 11 mutants unable to grow on 1,2epoxypropane. These 11 mutants were tested for growth in liquid medium containing 1,2-epoxypropane; three mutants did not grow. These mutants, designated M3, M8 and $\mathrm{M} 10$, all grew on acetone with growth rates comparable to wild-type Xanthobacter Py2.

\section{Characterization of mutants defective in epoxyalkane degradation}

The three mutants devoid of epoxyalkane-degrading activity were tested for the presence of the LMF. In wildtype Xanthobacter Py2 no epoxyalkane-degrading activity was found when cells were grown on yeast extractglucose medium; however, when $1 \%$ propene was added to the head-space during growth on yeast extract-glucose, low activities were observed [approx. 2 nmol 1,2epoxypropane degraded $\mathrm{min}^{-1}$ (mg protein $)^{-1}$. Crude extracts of mutant cells grown on yeast extract-glucose in the presence of $1 \%$ propene showed no epoxyalkanedegrading activity. These crude extracts were used for the preparation of the LMF and the protein fraction. By combining the protein fraction of the wild-type Py2 grown on 1.2-epoxypropane with the LMF of a mutant it is possible to detect whether the LMF is present in the mutant. The combination of the protein fraction of the wild-type Py2 with the LMF of the mutants resulted in epoxyalkane-degrading activity of between 4 and $6 \mathrm{nmol}$ 1,2-epoxyalkane degraded $\mathrm{min}^{-1}$ ( $\mathrm{mg}$ protein) ${ }^{-1}$. The specific activity of combined wild-type Xanthobacter Py2 LMF and protein fraction was 12 nmol 1,2-epoxyalkane degraded $\min ^{-1}$ (mg protein $)^{-1}$. No activity was detected when the LMF of the wild-type Xantbobacter Py2 was combined with the protein fraction of the mutant cells. These results show that the LMF is still present in the three mutants and that the protein fractions of the mutants are inactive.

\section{Complementation of Xanthobacter Py2 mutants}

The broad-host-range cosmid pLAFR 5 was used as a cloning vector for Xanthobacter Py2 because of its ability to replicate in Xanthobacter species (Janssen et al., 1989). The vector contains a double cos site, allowing the insertion of relatively large DNA fragments.

Screening of the gene bank with an average insert size of $17 \mathrm{~kb}$ was done by individually transferring the recombinant cosmids from the transduced E. coli LE392 to the recipient mutant $\mathrm{M} 10$ using triparental mating as described in Methods. The conjugated recipients were selected for growth on mineral medium plates in the presence of tetracycline using 1,2-epoxypropane as substrate. Nine tetracycline-resistant colonies were able to grow on the substrate. To rule out reversion of the mutation and to eliminate unstable cosmids, the nine cosmids were isolated from these complemented M10 colonies, used to transform E. coli TG1 and again conjugated into M10. Five out of nine cosmids (pEP1, pEP3, pEP4, pEP5 and pEP9) restored the ability to grow on 1,2-epoxypropane both on plates and in liquid mineral medium.

Restriction enzyme analysis of the five different cosmids with BamHI, XhoI, EcoRI and SalI revealed that the restriction pattern of $\mathrm{pEP} 3$ was identical to $\mathrm{pEP} 4$; the same was observed for $\mathrm{pEP} 5$ and $\mathrm{pEP}$, leaving three different cosmids ( $\mathrm{pEP} 1, \mathrm{pEP} 3$ and $\mathrm{pEP} 9$ ) complementing M10. The insert sizes of the three cosmids were 24,26 and $22 \mathrm{~kb}$ for $\mathrm{pEP} 1, \mathrm{pEP} 3$ and $\mathrm{pEP} 9$, respectively.

The three cosmids were conjugated into the mutants M3 and M8. They complemented M8 as seen from good growth on plates in the presence of 1,2-epoxypropane. The M3 mutant, however, was not complemented by the cosmids. A subsequent conjugation of 2200 clones from the gene bank into M3 did not yield any cosmid restoring its ability to grow on 1,2-epoxypropane. 


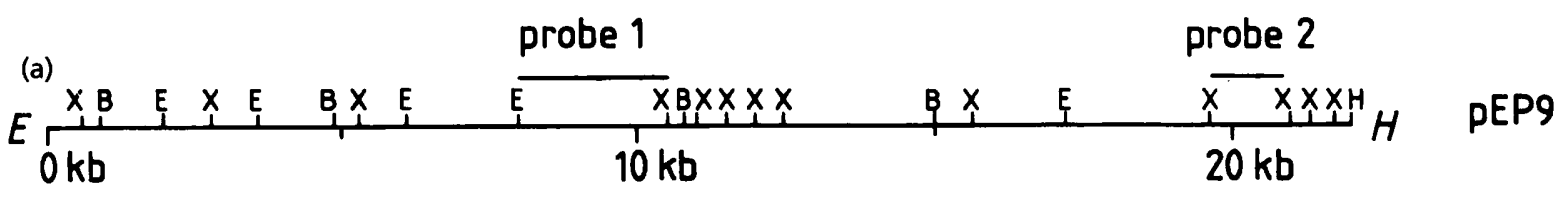

(b)<smiles>[CH][CH2]</smiles>

B

Fig. 2. Restriction map of the $22 \mathrm{~kb}$ insert of $\mathrm{pEP}$. The lines at the top represent the probes used for Southern hybridization with pEP1, pEP3 and pEP9 (Fig. 3). Restriction enzymes: B, BamHI; E, EcoRI; X, Xhol; H, HindlII. The sites for restriction enzymes in italics belong to the multiple cloning site of the vector. (b) Deletion clones of pEP9. pEP9.1 is a BamHI and pEP9.2 a EcoRI deletion clone.

\section{Epoxyalkane-degrading activity in complemented mutant cells}

Epoxyalkane-degrading activity was measured in crude extracts of mutants M8 and M10 grown under different conditions. The activity of cells grown on 1,2epoxypropane was comparable to that of wild-type Xanthobacter Py2 $\left.\left[13 \pm 2 \mathrm{nmol} \mathrm{m^{-1 }} \text { (mg protein }\right)^{-1}\right]$. Surprisingly, the M8 and M10 mutants complemented with $\mathrm{pEP} 9$ showed a high epoxyalkane-degrading activity of $22 \mathrm{nmol} \mathrm{min}{ }^{-1}$ ( $\mathrm{mg}$ protein) $)^{-1}$ when grown on yeast extract-glucose medium in the absence of 1,2epoxypropane, indicating constitutive expression. This was not observed in wild-type Xanthobacter Py 2 cells nor in the mutants M8 and M10 complemented with pEP1 or pEP3.

\section{Cofactor-dependent epoxyalkane-degrading activity in $X$. autotrophicus GJ10}

The plasmid pEP9, which constitutively expressed epoxyalkane-degrading activity in M8 and M10, was conjugated into $X$. autotrophicus GJ10. This Xanthobacter strain is not able to grow on epoxyalkanes. Conjugants were selected on mineral medium plates containing tetracycline and 2-propanol. The conjugated $X$. autotrophicus G J10 was not able to grow on 1,2-epoxypropane and no epoxyalkane-degrading activity was detected in crude extracts of cells grown on yeast extract-glucose. However, 1,2-epoxypropane was degraded by these extracts [2 nmol 1,2-epoxypropane degraded $\mathrm{min}^{-1}$ ( $\mathrm{mg}$ protein $)^{-1}$ ] if the LMF prepared from propene-grown Xanthobacter Py 2 cells was added. No such activity was found when the LMF was added to crude extracts of wildtype $X$. autotropbicus GJ10.

\section{Restriction map of pEP9 and hybridization studies with PEP1 and PEP3}

Because pEP9 carries the information for epoxyalkanedegrading activity, as seen from $X$. autotrophicus GJ10 conjugated with pEP9, this cosmid was further charac- (a)

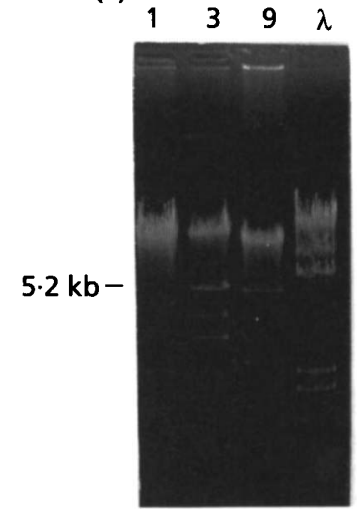

(b)

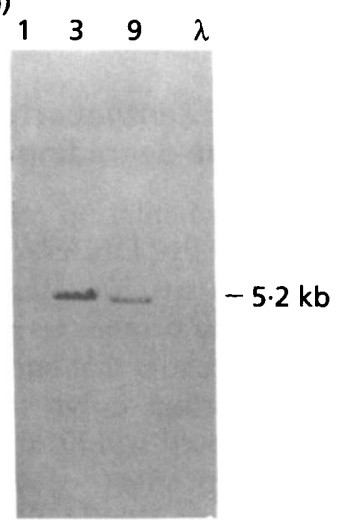

(c)

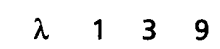

(d)

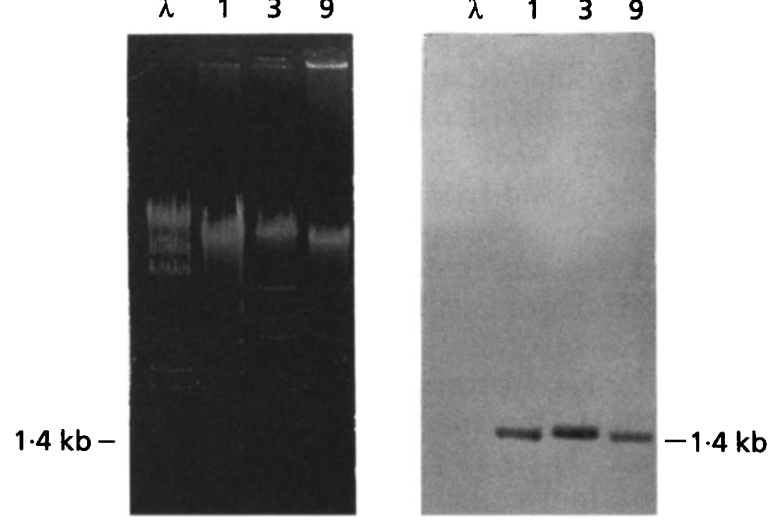

Fig. 3. Southern hybridization studies of Xhol-digested pEP1 (lane 1), PEP3 (lane 3) and pEP9 (lane 9). (b) is the blot of digest (a) hybridized with probe 1 (Fig. 2). (c) and (d) are the digest and the blot hybridized with probe 2 . A $\lambda / H$ HindIII marker was used.

terized. A restriction map of $\mathrm{pEP} 9$ was constructed based on migration patterns of digested $\mathrm{pEP} 9$ and on Southern hybridization of digests of $\mathrm{pEP} 9$ with several probes from the pEP9 insert (Fig. 2). 
Southern hybridization of XboI digests of pEP1, pEP3 and $\mathrm{pEP} 9$ are shown in Fig. 3. The blots were hybridized to two different probes of pEP9 (Fig. 2). Probe 1 is a $2.5 \mathrm{~kb}$ EcoRI-XhoI fragment (from $8 \mathrm{~kb}$ to $10.5 \mathrm{~kb}$ on the map) and probe 2 is a $1.4 \mathrm{~kb}$ XhoI fragment (from $19.6 \mathrm{~kb}$ to $21 \mathrm{~kb}$ on the map). Probe 1 hybridized only to a $5.2 \mathrm{~kb}$ $X$ hoI fragment of $\mathrm{pEP} 3$, whereas probe 2 hybridized to both pEP1 and pEP3. Further restriction analyses with XboI (Fig. 3), EcoRI, SalI and BamHI showed that pEP3 is homologous to pEP9 from approximately $5 \mathrm{~kb}$ to $22 \mathrm{~kb}$ on the pEP9 map. The cosmid pEP1 showed considerably less homology with both $\mathrm{pEP} 3$ and $\mathrm{pEP} 9$. Restriction homology with XhoI (Fig. 3) and SalI was only found from the $18 \mathrm{~kb}$ point to approximately the $21.5 \mathrm{~kb}$ point of cosmid pEP9.

\section{Subcloning of pEP9}

The homology studies with pEP1 in particular indicated that the complementing area was present on the righthand end of pEP9 (Fig. 3). Therefore, this part of pEP9 was used to construct subclones. Cosmid $p E P 9$ was digested with $B a m \mathrm{HI}$ or $E c o \mathrm{RI}$ and religated to delete the internal fragments. The resulting cosmids were checked by restriction analysis and assayed for complementation of mutant M10. The Bam HI and the EcoRI deletion clones of pEP9, designated pEP9.1 and pEP9.2, respectively, had the ability to complement mutant M10 (Fig. 2). The nucleotide sequence of the complementing $4.8 \mathrm{~kb}$ EcoRI-HindIII fragment of pEP9.2 was determined.

\section{Nucleotide sequence of the $4.8 \mathrm{~kb}$ complementing fragment of pEP9.2}

The nucleotide sequence is presented in Fig. 4. The G $+C$ content of the fragment was $65.4 \mathrm{~mol} \%$, which is lower than the $70 \mathrm{~mol} \%$ found in Xanthobacter Py2 (Van Ginkel \& De Bont, 1986). Four different ORFs were found, using the codon preference normally observed for Xantbobacter DNA. The four ORFs code for proteins of $41690,7388,57315$ and $26111 \mathrm{Da}$, respectively. In the opposite strand no ORFs of significant length were found.

A computer search of the Swiss-Prot protein bank did not reveal homology for the first two ORFs. But, interestingly, ORF3 showed significant homology with mercury(II) reductase (EC 1.16.1.1) from Bacillus strain RC607 (P16171), glutathione reductase (NADPH) (EC 1.6.4.2) from Pseudomonas aeruginosa (P23189) and dihydrolipoamide dehydrogenase (EC 1.8.1.4) from pig (P09623). For ORF4, homologies were found with 3oxoacyl reductase (EC 1.1.1.35) from Cuphea lanceolata (P28643) and glucose 1-dehydrogenase (EC 1.1.1.47) from Bacillus subtilis (P12310).

\section{DISCUSSION}

Three major problems are encountered concerning the isolation of the gene(s) responsible for epoxyalkane degradation in Xanthobacter Py2. The first is the absence of a purified enzyme (Weijers et al., 1994). The second is the low specific activity of the enzyme: in crude extracts with 1,2-epoxypropane as substrate epoxyalkane-degrading activity was only $13 \mathrm{nmol} \mathrm{\textrm {min } ^ { - 1 }}$ (mg protein) ${ }^{-1}$. With trans-2,3-epoxybutane, which is degraded enantioselectively, this activity is even lower $\left[ \pm 1 \mathrm{nmol}\right.$ per $\mathrm{min}^{-1}$ $(\mathrm{mg} \text { protein })^{-1}$. Consequently, 1,2-epoxypropane was chosen as the model substrate to monitor epoxidedegrading activity. The third problem is the cofactor requirement in epoxyalkane degradation. Apart from NAD, another unidentified factor is necessary for activity. This cofactor requirement makes the isolation of the epoxyalkane-degrading protein or expression of the cloned genes in other strains more difficult. Recently it has been demonstrated that DTT can replace the unknown cofactor (Weijers et al., 1994). However, in the present study the low molecular mass fraction (LMF) of crude cell extracts which contain the cofactor has been used rather than DTT to avoid any possible artefacts.

In view of the above difficulties, it was decided to isolate mutants lacking epoxyalkane-degrading activity which were subsequently used to isolate by complementation the gene(s) involved in epoxyalkane degradation. The procedure for creating and selecting mutant Xanthobacter strains was successful: the three mutants selected met all the requirements for use in complementation studies. They were unable to grow on 1,2-epoxypropane, but grew on acetone, the product of 1,2-epoxypropane degradation, and they contained the LMF.

Three different cosmids were found to restore the ability of mutant M10 to grow on 1,2-epoxypropane. These cosmids were also able to complement mutant M8. The epoxyalkane-degrading activities of the complemented mutants M8 and M10 were comparable to the wild-type Xanthobacter Py2. The finding that mutant M3 was not complemented by the cosmids suggests that it belongs to a complementation group different from M8 and M10. However, the inability to complement this mutant with the Xantbobacter Py2 gene bank shows that the M3 mutation is more complex.

Complementation of mutants lacking epoxyalkane-degrading activity does not itself necessarily imply that the structural genes for the degrading enzymes have been cloned. To determine whether this was the case cosmid pEP9 was conjugated into $X$. autotrophicus GJ10. Although $X$. autotropbicus GJ10(pEP9) did not grow on 1,2-epoxypropane, the protein required for epoxyalkanedegrading activity was present in extracts of cells grown on yeast extract-glucose. This was demonstrated by combining LMF obtained from wild-type Xanthobacter Py2 with the protein fraction of $X$. autotrophicus GJ10(pEP9), which resulted in 1,2-epoxypropane degradation and acetone formation.

The ORF(s) encoding the protein(s) involved in epoxyalkane degradation remain to be identified. The epoxidedegrading enzyme has been characterized only to a limited extent, but a reaction mechanism has been proposed (Weijers et al., 1994). The mechanism suggested involves 
AAGCTTCGCTCCAGGTCGACGATCAAGAAAATAGGTECGGAAGCTTTCCCACTGCTCGGCGGGATTGCGGTAATGCAGGCAGATGTGGCEATEAAGAG

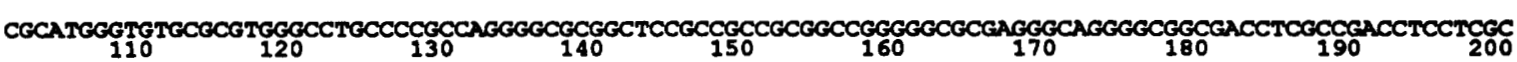

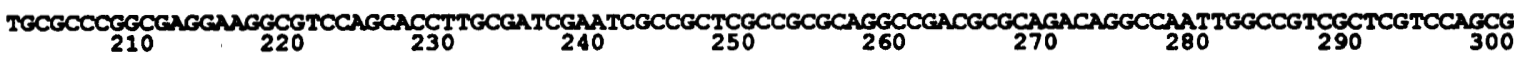

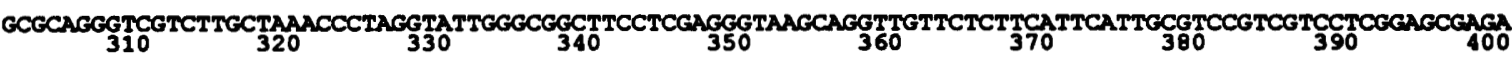

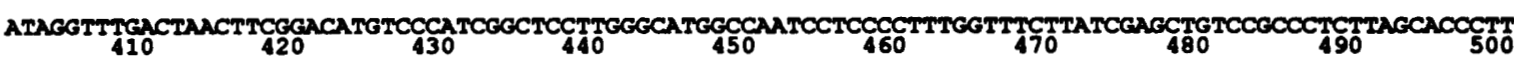

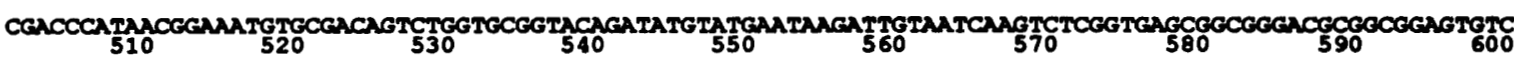

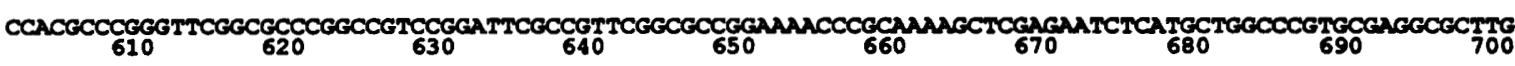

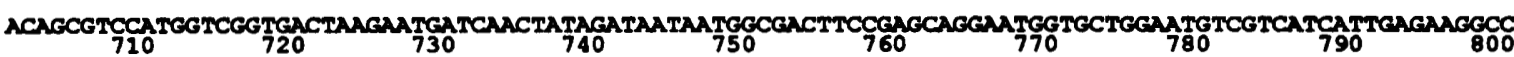

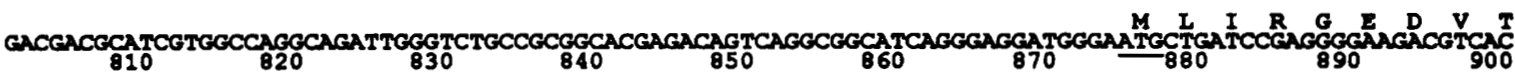

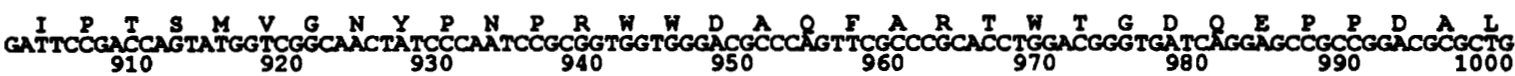

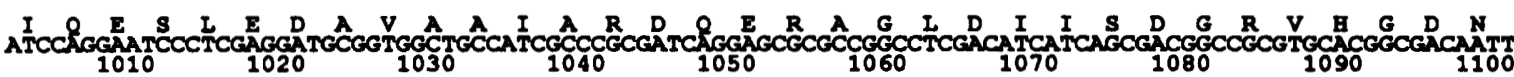
Y.A A

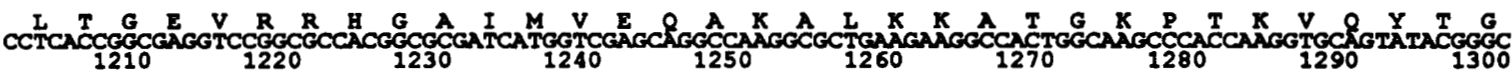

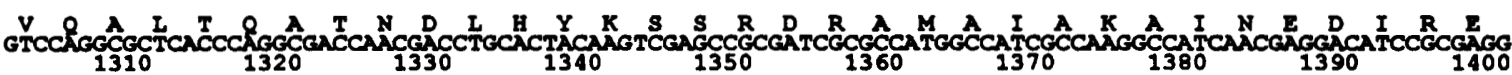

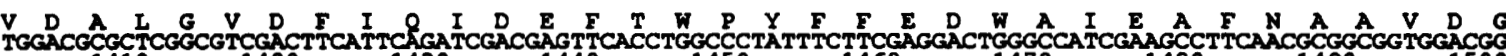

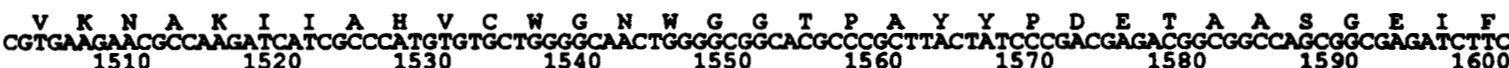

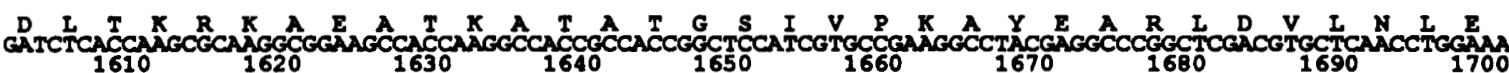

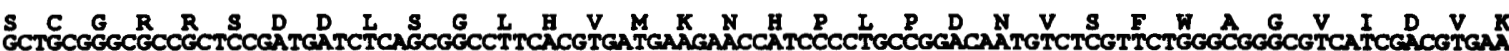
$\begin{array}{llllllllll}1710 & 1720 & 1730 & 1740 & 1750 & 1760 & 1770 & 1780 & 1790 & 1800\end{array}$

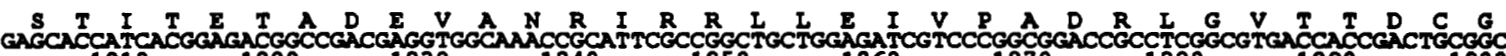

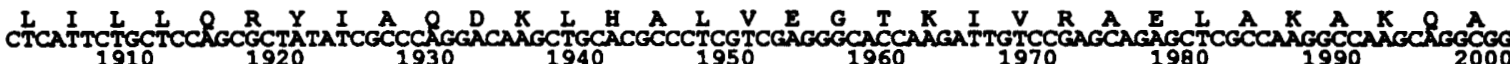

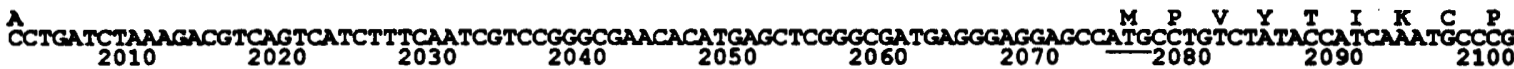

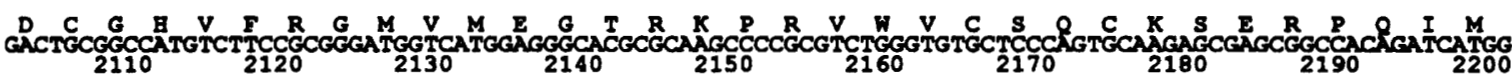

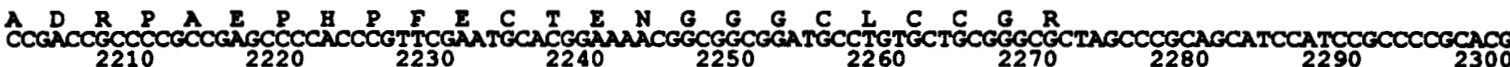

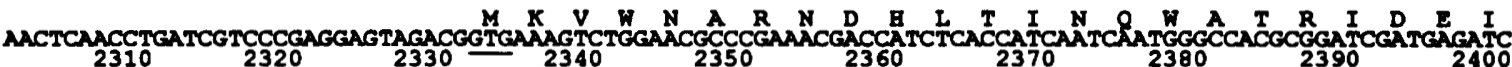

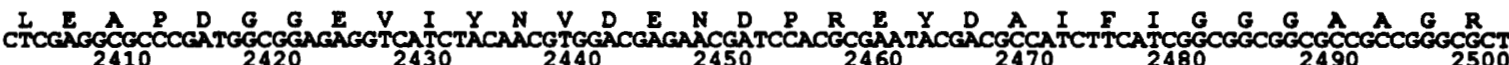
TCCGCTCCGCCTATCTGCGCGCCATGGECGGCCGGCAGCTCATCGTCGACCECTGGCCGTTCCTGGGCGGCTCGTGCCCGCACAATGCGTGCGTGCCGCA

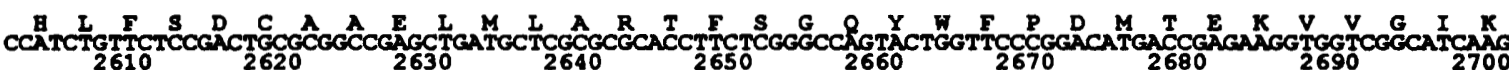

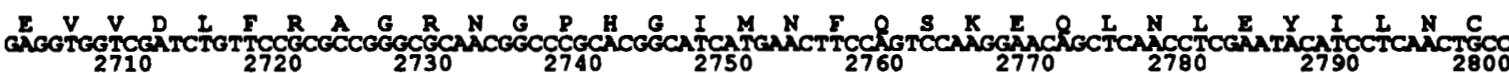

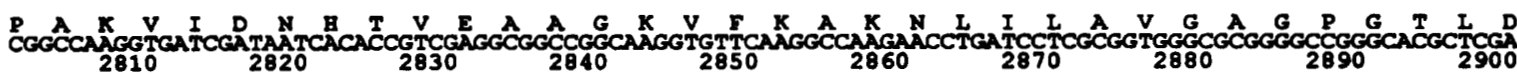

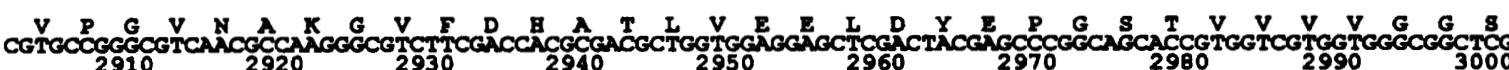

Fig. 4. For legend see facing page. 


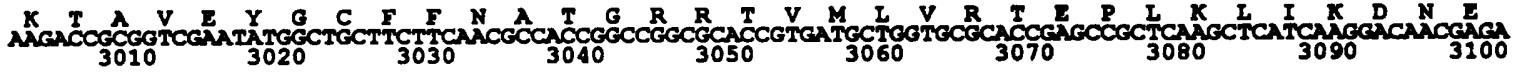

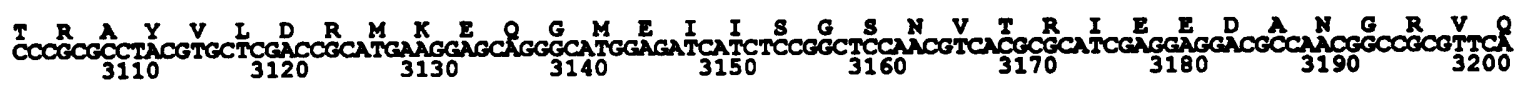

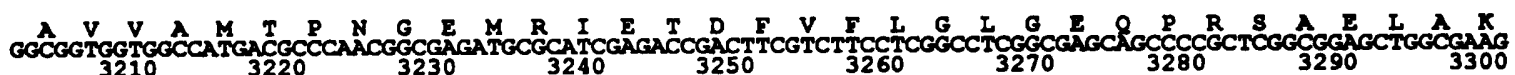

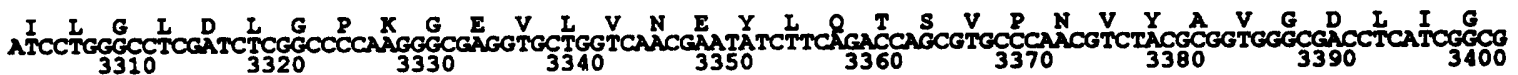

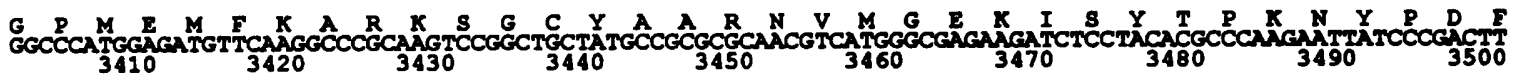

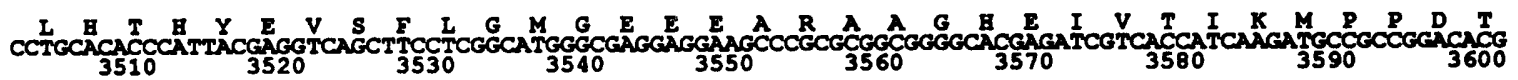

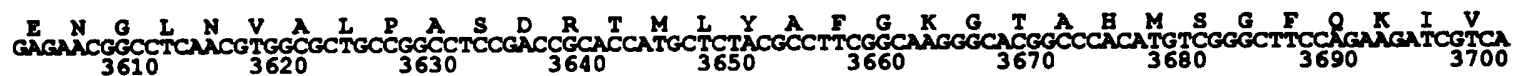

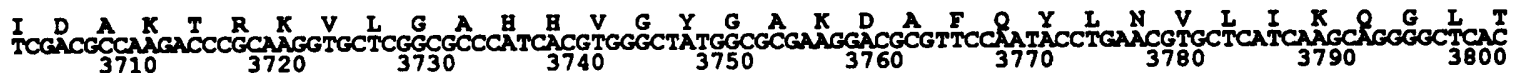

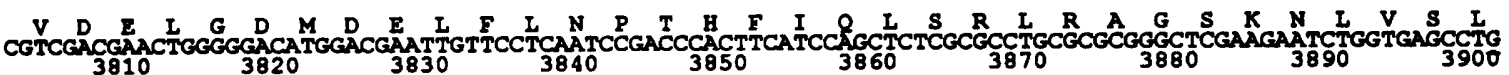

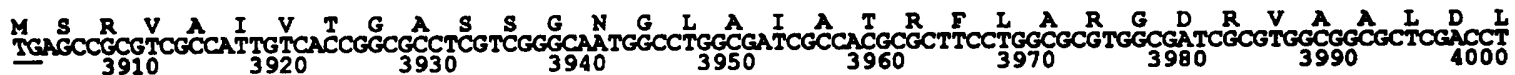

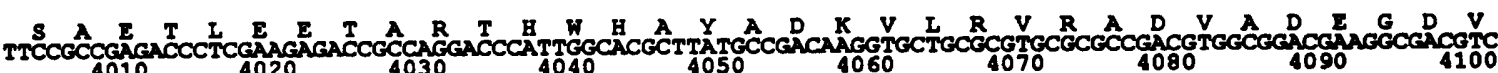

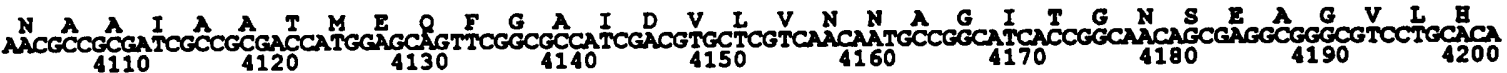

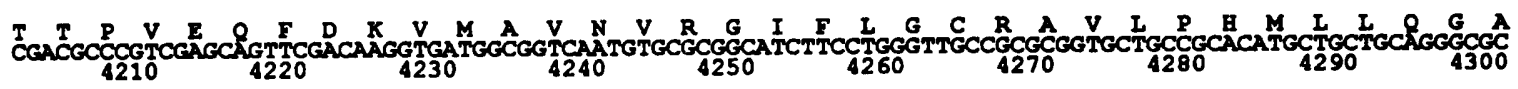

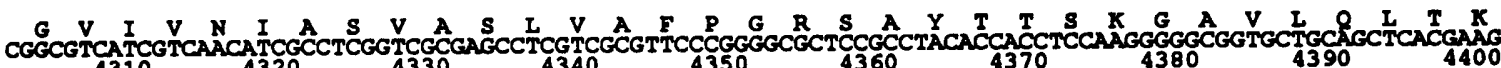

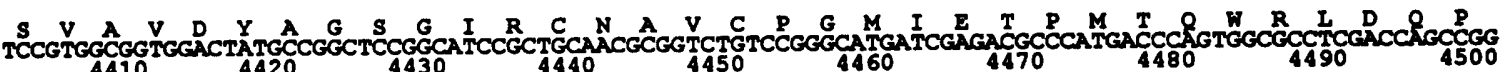

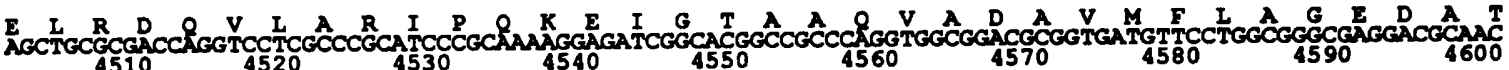

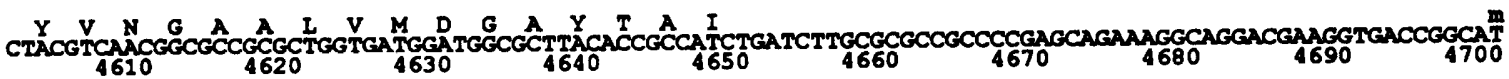

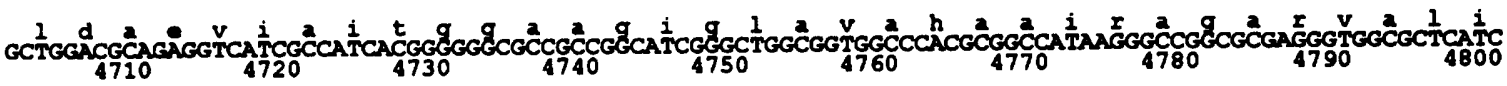

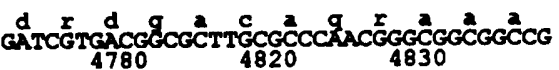

Fig. 4. Nucleotide sequence of the $4.8 \mathrm{~kb}$ EcoRl-Hindlll fragment complementing the Xanthobacter Py2 M10 mutant deficient in epoxyalkane-degrading activity, and the deduced amino acid sequence of ORF1 (nt 875-2002), ORF2 (2074-2271), ORF3 (2332-3900) and ORF4 (3900-4649). The start codon of each ORF is underlined. The nucleotide sequence is presented from the HindIII to the EcoRI site.

the reduction of a disulfide bridge in the catalytic centre of the enzyme. Similarities were noted with vitamin K epoxide reductase and very recently the involvement of lipoamide reductase was implied in supplying reducing equivalents (Thyssen et al., 1994). It is therefore very interesting that ORF3 shows significant homology with lipoamide reductase.

\section{ACKNOWLEDGEMENTS}

We thank Professor Dr D. B. Janssen for providing helpful advice and for supplying strains and plasmids. We would like to thank Dr D. Leak and Professor M. Fonseca and their coworkers for interesting discussions in the framework of our joint EC project. This study was supported by a grant from the European Community (BIOT-CT91-0269).

\section{REFERENCES}

Boyer, H. W. \& Roulland-Dussoix, D. (1969). A complementation analysis of the restriction and modification of DNA in Escherichia coli. J Mol Biol 41, 459-472.

De Bont, J. A. M. (1993). Biotransformation of optically pure epoxides. Tetrabedron Asymmetry 4, 1331-1340.

Figurski, D. H. \& Helinski, D. R. (1979). Replication of an origincontaining derivative of plasmid RK2 dependent on a plasmid function provided in trans. Proc Natl Acad Sci US A 76, 1648-1652. Janssen, D. B., Scheper, A., Dijkhuizen, L. \& Witholt, B. (1985). Degradation of halogenated aliphatic compounds by Xanthobacter autotrophicus GJ10. Appl Environ Microbiol 49, 673-677.

Janssen, D. B., Pries, F., Van Der Ploeg, J., Kazemier, B., Terpstra, P. \& Witholt, B. (1989). Cloning of 1,2-dichloroethane degradation 
genes of Xanthobacter autotrophicus GJ10 and expression and sequencing of the $d b l A$ gene. $J$ Bact 171, 6791-6799.

Keen, N. T., Tamaki, S., Kobayahi, D. \& Trollinger, D. (1988). Improved broad-host-range plasmids for DNA cloning in Gramnegative bacteria. Gene 70, 191-197.

Leak, D. J., Aikens, P. J. \& Seyed-Mahmoudian, M. (1992). The microbial production of epoxides. Trends Biotechnol 10, 256-261.

Sambrook, J., Fritsch, E. F. \& Maniatis, T. (1989). Molecular Cloning: a Laboratory Manual, 2nd edn. Cold Spring Harbor, NY: Cold Spring Harbor Laboratory.

Sanger, F., Nicklen, S. \& Coulsen, A. R. (1977). DNA sequencing with chain-terminating inhibitors. Proc Natl Acad Sci USA 74, 5463-5467.

Thijssen, H. H. W., Janssen, Y. P. G. \& Vervoort, L. T. M. (1994). Microsomal lipoamide reductase provides vitamin $\mathrm{K}$ epoxide reductase with reducing equivalents. Biochem J 297, 277-280.

Van Ginkel, C. G. \& De Bont, J. A. M. (1986). Isolation and characterization of alkene-utilizing Xantbobacter spp. Arch Microbiol 145, 403-407.

Weijers, C. A. G. M., De Haan, A. \& De Bont, J. A. M. (1988a). Chiral resolution of 2,3-epoxyalkanes by Xantbobacter Py2. Appl Microbiol Biotechnol 27, 337-340.

Weijers, C. A. G. M., De Haan, A. \& De Bont, J. A. M. (1988b). Microbial production and metabolism of epoxides. Microbiol $S_{c i} \mathbf{5}$, 156-159.

Weijers, C. A. G. M., Jongejan, H., Franssen, M. C. R., De Groot, Ae. \& De Bont, J. A. M. (1994). Dithiol- and NAD-dependent degradation of epoxyalkanes by Xanthobacter Py2. Appl Microbiol Biotechnol (in press).

Wiegant, W. M. \& De Bont, J. A. M. (1980). A new route for ethylene glycol metabolism in Mycobacterium E44. J Gen Microbiol 120, 325-331.

Received 1 July 1994; revised 11 August 1994; accepted 18 October 1994. 